EPJ Web of Conferences 41, 11006 (2013)

DOI: $10.1051 /$ epjconf/20134111006

(C) Owned by the authors, published by EDP Sciences, 2013

\title{
High Brightness XUV Frequency Combs via Intracavity High Harmonic Generation
}

\author{
T. K. Allison, ${ }^{1}$ A. Cingöz, ${ }^{1}$ C. Benko, ${ }^{1}$ D. C. Yost, ${ }^{1}$ A. Ruehl, ${ }^{2}$ M. Fermann, ${ }^{2}$ I. Hartl, ${ }^{2}$ and J. $\mathrm{Ye}^{1}$ \\ 1 JILA, National Institute of Standards and Technology and University of Colorado, Boulder, CO \\ 80309-0440, USA \\ 2 IMRA America Inc., 1044 Woodridge Avenue, Ann Arbor, Michigan 48105, USA
}

\begin{abstract}
We report the generation of high power extreme ultraviolet frequency combs at $154 \mathrm{MHz}$ repetition rate. The XUV combs are characterized by conducting high resolution spectroscopy and observing the heterodyne beats between two independent systems.
\end{abstract}

\section{Introduction}

We report on recent advances made in our group [1-3] on XUV frequency comb generation via intracavity high order harmonic generation (HHG). Since HHG is initiated through field ionization, it is typically realized with low repetition rate $(<100 \mathrm{kHz})$ amplified femtosecond laser systems producing high energy pulses $(>100 \mu \mathrm{J})$, with average powers up to tens of Watts. In an alternative method, the modes of a frequency comb and a high-finesse external cavity are locked, leading to $>100 \mathrm{MHz}$ repetition rates and multi-kW average powers, while still supporting peak intensities sufficient for HHG. However, in the initial demonstrations $[4,5]$, the generated XUV power did not increase commensurately, and the frequency comb structure in the harmonic light was not verified for the above-threshold harmonics in the XUV region.

Through advances in high power Yb:fiber frequency combs [6], an efficient and robust output coupling scheme [7], and a deep understanding of the nonlinear optical response of the cavity/HHG plasma system [2,3], we have demonstrated the generation of more than $200 \mu \mathrm{W} /$ harmonic, among the highest HHG yields we are aware of [8,3]. Further, we have confirmed the frequency comb structure in the XUV by conducting direct frequency comb spectroscopy at $82 \mathrm{~nm}$ and $63 \mathrm{~nm}$ [1], simultaneously demonstrating a spectroscopic instrument with unrivaled resolution in the XUV. We will discuss these results and further investigation of the coherence of the generated XUV combs by recording heterodyne beats between harmonics from two independent intracavity HHG systems. These heterodyne beats will allow examination of the phases of the XUV light produced through HHG with unprecedented precision.

\section{Intracavity HHG Source}

Our intracavity HHG source is shown in figure 1. A high-power Yb:fiber frequency comb [6] centered at $1070 \mathrm{~nm}$ with a repetition rate of $154 \mathrm{MHz}$ is used to resonantly excite a five mirror femtosecond enhancement cavity with a power buildup factor of approximately 200. Xenon gas is introduced with a 100-200 $\mu \mathrm{m}$ glass nozzle at an intracavity focus formed between two concave mirrors with radii of curvature of $10 \mathrm{~cm}$ and $15 \mathrm{~cm}$. The focus size (FWHM) is estimated to be $29 \mu \mathrm{m}$ (horizontal) $\times 17 \mu \mathrm{m}$ (vertical). The XUV light is diffracted out of the cavity with the hybrid grating/dielectric mirror output coupler described by Yost et al. [7].

This is an Open Access article distributed under the terms of the Creative Commons Attribution License 2.0, which permits unrestricted use, distribution, and reproduction in any medium, provided the original work is properly cited. 


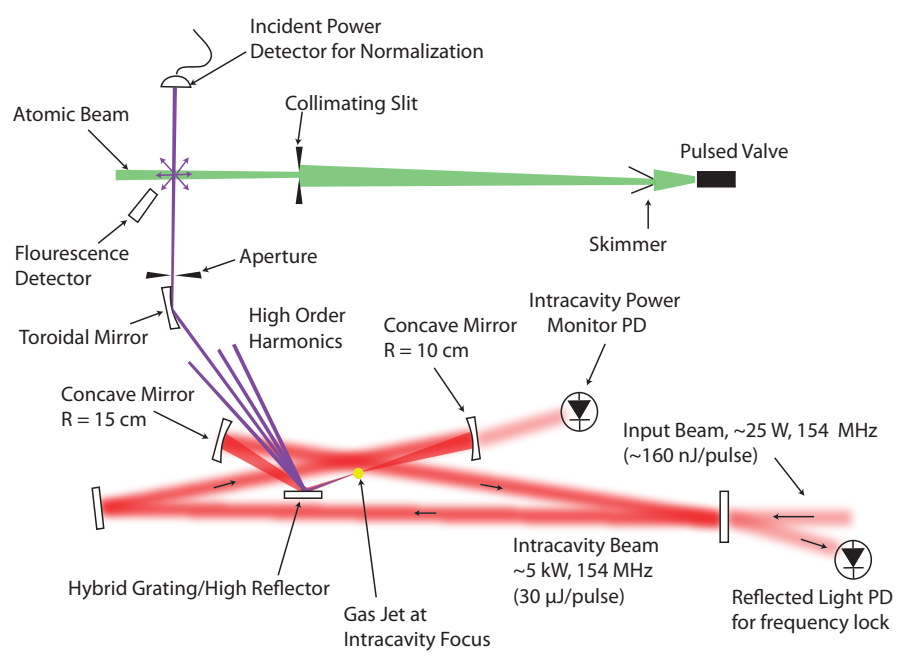

Fig. 1. Intracavity HHG source and direct frequency comb spectroscopy apparatus. XUV power measurements are made by inserting an XUV photodiode and a sodium salicylate coated plate before the toroidal mirror.

\section{Power Measurements}

The $13^{\text {th }}$ harmonic power is measured with a Si XUV photodiode coated with indium (IRD Inc. AXUV100In/ $/ \mathrm{MgF}_{2}$ ), and was confirmed to be insensitive to scattered IR and $3^{\text {rd }}$ harmonic light in the present geometry. The intensity of the other harmonics relative to the $13^{\text {th }}$ harmonic is measured by recording an image of the dispersed harmonic light on a sodium salicylate coated plate. A typical image is shown in the top of figure 2. The HHG spectra is obtained by vertical integration of the image. An optimized spectrum is shown in figure 2. The measured outcoupled power exceeds 20 $\mu \mathrm{W} /$ harmonic. Since the grating has an estimated diffraction efficiency of less than $10 \%$, this corresponds to more than $200 \mu \mathrm{W} /$ harmonic generated at the intracavity focus.

\section{Temporal Coherence}

To demonstrate the comb structure of the XUV radiation, we have spectrally resolved the $3 s^{2} 3 p^{6} \mathrm{~J}=0$ $\rightarrow 3 \mathrm{~s}^{2} 3 \mathrm{p}^{5} 5 \mathrm{~d} \mathrm{~J}=1$ electric dipole (E1) transition in argon at $82 \mathrm{~nm}\left(13^{\text {th }}\right.$ harmonic) and the $2 \mathrm{~s}^{2} 2 \mathrm{p}^{6} \mathrm{~J}=0$ $\rightarrow 2 \mathrm{~s}^{2} 2 \mathrm{p}^{5} 4 \mathrm{~s} \mathrm{~J}=1 \mathrm{E} 1$ transition at $63 \mathrm{~nm}\left(17^{\text {th }}\right.$ harmonic) in neon by means of resonance fluorescence spectroscopy. For direct frequency comb spectroscopy, the XUV photodiode and salicylate coated plate are removed and the beam is instead collimated with a toroidal mirror and directed to intersect a pulsed supersonic expansion of Ar or Ne gas. Fluorescence photons from the atomic beam are detected with a photomultiplier after conversion to $420 \mathrm{~nm}$ via a second sodium salicylate phosphor.

The Doppler width of the resolved atomic transitions can be narrowed by closing a slit at the end of the atomic beam source. In figure 2c), we have narrowed the Ar transition Doppler width to $\sim 10 \mathrm{MHz}$ and there is no evidence of the comb linewidth contributing to the observed width of the transition, indicating an upper bound for the XUV comb tooth linewidth of less than $10 \mathrm{MHz}$.

To further test the temporal coherence of the XUV comb source, we have constructed a system consisting of two identical intracavity HHG XUV comb sources. Light from the Yb:fiber comb is split with an acousto-optic modulator to produce two near-infrared combs whose carrier envelope offset frequencies, $f_{0}$, differ by several MHz. These two near-infrared combs excite two separate intracavity HHG systems to produce XUV combs with $f_{0}$ 's offset from each other. The XUV combs are then combined on a photodetector to produce beat notes in the radio frequency domain. 

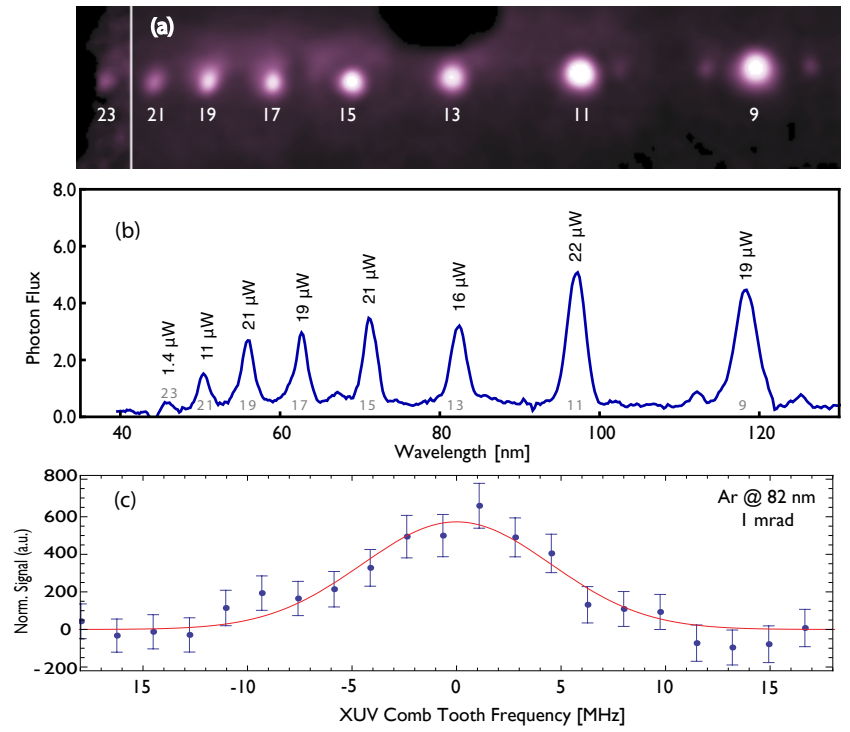

Fig. 2. a) Image of the dispersed harmonics recorded on the sodium salicylate plate. b) HHG spectrum. The outcoupled power in each harmonic is indicated above each harmonic peak. c) Scan of a single XUV comb tooth across the Ar transition frequency.

\section{References}

1. A. Cingöz, D. C. Yost, T. K. Allison, A. Ruehl, M. E. Fermann, I. Hartl, and J. Ye, "Direct frequency comb spectroscopy in the extreme ultraviolet," Nature 482, 68-71 (2012).

2. T. K. Allison, A. Cingöz, D. C. Yost, and J. Ye, "Extreme nonlinear optics in a femtosecond enhancement cavity," Phys. Rev. Lett. 107, 183903 (2011).

3. D. C. Yost, A. Cingöz, T. K. Allison, A. Ruehl, M. E. Fermann, I. Hartl, and J. Ye, "Power optimization of xuv frequency combs for spectroscopy applications [invited]," Opt. Express 19, 2348323493 (2011).

4. R. J. Jones, K. D. Moll, M. J. Thorpe, and J. Ye, "Phase-coherent frequency combs in the vacuum ultraviolet via high-harmonic generation inside a femtosecond enhancement cavity," Physical Review Letters 94, 193201 (2005).

5. C. Gohle, T. Udem, M. Herrmann, J. Rauschenberger, R. Holzwarth, H. A. Schuessler, F. Krausz, and T. W. Hänsch, "A frequency comb in the extreme ultraviolet," Nature 436, 234-237 (2005).

6. A. Ruehl, A. Marcinkevicius, M. E. Fermann, and I. Hartl, "80 w, 120 fs yb-fiber frequency comb," Opt. Lett. 35, 3015-3017 (2010).

7. D. C. Yost, T. R. Schibli, and J. Ye, "Efficient outpult coupling of intracavity high harmonic generation," Opt. Lett. 33, 1099-1101 (2008).

8. J. Lee, D. R. Carlson, and R. J. Jones, "Optimizing intracavity high harmonic generation for xuv fs frequency combs,” Opt. Express 19, 23315-23326 (2011). 